\title{
La producción jus filosófica de Norberto Bobbio y la enseñanza del Derecho para sociedades auténticamente democráticas
}

\section{The legal Philosophical Production of Nor- berto Bobbio and the Teaching of the Law in Authentically Democratic Societies}

\author{
William Meza Arala *
}

Fecha de recepción: 29 de abril de 2008 Fecha de aprobación: 5 de mayo de 2008

\section{Resumen}

La comprensión acerca de las relaciones comunicativas de la tríada: persona, sociedad, Derecho, propia del Módulo Introductorio, encuentra una adecuada interpretación en la Teoría General del Derecho aludida. La Teoría del Ordenamiento Jurídico, es concluyente acerca de las explicaciones obtenidas del comportamiento de la pluralidad de normas jurídicas derivadas de la Teoría de la Norma Jurídica. Se aclara el funcionamiento sistémico en la producción de justicia, en la que intervienen de manera ordenada varios de sus operadores. Finalmente, la explicación del Derecho, como sistema, permite aclarar de manera metódica y pedagógica la relación entre poderes y deberes del Estado Social de Derecho y Derechos Humanos.

\section{Palabras clave}

Teoría del derecho, enseñanza del derecho, derecho y democracia 


\begin{abstract}
The understanding of the relations of the triad person - society - law finds an appropriate interpretation in Bobbio's General Theory of Law. His Theory of Legal Order is conclusive with regard to the explanations of the behaviour of the plurality of legal norms (explanations that derive from the Theory of the Legal Norm). It clarifies the systematic functioning of the production of law, in which a variety of its operators intervene in an organized way. The explanation of the law as a system allows the clarification, in a methodological and pedagogical way, of the relation between the powers and the duties of a social constitutional state.
\end{abstract}

\title{
Key words
}

Theory of law, teaching of law, law and democracy

\section{INTRODUCCIÓN}

Con base en mi práctica pedagógica del Derecho, por más de treinta años y en diversas universidades, puedo confirmar la importancia de enseñar Derecho mediante el uso de los enfoques de esa Escuela Italiana, la cual, en gran parte, ha sido liderada por Norberto Bobbio, a finales del siglo XX. Gracias a esta escuela ha habido grandes cambios recientes en los enfoques ideológicos, como por ejemplo, la desaparición de los dogmatismos de las extremas hegelianas de los siglos XIX y XX, lo cual ha traído como consecuencia la reivindicación de la enseñanza del Derecho como ciencia de la libertad y como medio de realización del progreso humano social, por la vía de la democracia como auténtica vía de comunicación y de socialización local y global de valores, claramente alcanzados por la humanidad entera.

La obra fundamental de dicho autor, Teoría general del Derecho, hace que surjan inquietudes interesantes en relación con el uso más apropiado del Derecho y de la democracia, ya que Colombia, como sociedad en vía de desarrollo, padece de muchos de los defectos de los cuales también han sufrido sociedades hoy en día desarrolladas, como es el hecho de caer en el defecto grave de los apasionamientos propios de una educación maniquea, gran parte culpable de la violencia que todavía malogra las buenas intenciones de paz y de prosperidad.

\section{LA CONSTITUCIÓN DE 1991 PRETEXTO DE NUEVOS Y PROVECHOSOS ENFOQUES DEL DERECHO EN TODOS LOS CENTROS DEL PENSAMIENTO COLOMBIANO}

En 1991, en plena etapa de la reforma constitucional colombiana y a raíz de las discusiones que se daban en la Corte Suprema de Justicia, en ese momento, así como en los centros educativos de enseñanza superior con respecto al llamado "nuevo Derecho", tuve la posibilidad de conocer el libro de Norberto Bobbio, Teoría general del Derecho, gracias a algunos magistrados que se actualizaban en los nuevos enfoques.

En el Prólogo a la primera edición castellana de 1992, Bobbio agradece al Dr. Rozo Acuña su traducción del italiano, la cual fue corregida y perfeccionada posteriormente por el traductor Doctor Jorge Guerrero, texto que recoge dos cursos universitarios del mencionado maestro: Teoría de la Norma Jurídica (1958) y Teoría del Ordenamiento Jurídico (1960). Estos dos trabajos que son el producto de una verdadera actualización investigativa del Derecho; su importancia radica en que quienes han sido formados en las escuelas colombianas repiten las enseñanzas que les dieron, las cuales están basadas en textos desactualizados, sobre todo, porque hacen referencia a procesos no integrados ni dinamizados por las metodologías 
integrativas, producto de la investigación ínter y multi disciplinaria, de la cual se ocupan los más avanzados científicos del Derecho colombiano.

\section{LA ACREDITACIÓN DE CENTROS DOCENTES Y SU ACTUALIZACIÓN}

El análisis del enfoque de la Escuela Italiana es pertinente. La Facultad de Derecho de la Universidad Santo Tomás, desde la acreditación de sus programas, se ha empeñado en actualizar permanentemente su enseñanza modular, la cual, mediante un esfuerzo de investigación constante, propende a que sus módulos se encuentren actualizados, que traten autores que no sólo estén acordes con sus orientaciones humanísticas y los perfiles profesionales que se buscan a través del PEI, sino que también inculquen en el estudiante los valores supremos de la democracia.

Es evidente que el Derecho no puede seguir siendo enseñado como disciplina autosuficiente, desconectada de las demás Ciencias Sociales, incluyendo los avances investigativos de la matemática, especialmente de los avances de la lógica, ni mucho menos de las Ciencias Socio-Lingüísticas (Semiótica, Pragmática, Gramática del texto, la Lingüística funcional, el Estructuralismo, etc.) (Van Dijk, 1989; Cárdenas \& Beltrán, 1990), que con el avance de las comunicaciones ofrecen a la operatividad del Derecho innovaciones verdaderamente sorprendentes, para agilizar los procesos judiciales, así como para la demanda de una justicia que marche a tono con el respeto a los Derechos Humanos y simultáneamente al desarrollo de una democracia plena.

Como bien se destaca en el mencionado Prólogo a la primera edición castellana, Bobbio reconoce el aporte efectuado por Francesco Carnelutti en Teoría general del Derecho -aparecida en 1946-. En este texto se ordena, en un sistema coherente y conceptualmente riguroso, la materia variada y compleja del derecho positivo italiano, surgido en muchas ocasiones sin un proyecto ordenador preciso. En este avance investigativo se mostraba la necesidad de que la teoría general del Derecho se diferenciara, tanto de la filosofía del Derecho como de la Ciencia Jurídica. Lo anterior era la lectura que Kelsen había hecho del Derecho y de la justicia, en años anteriores con gran acogida en Italia; con base en esa lectura y que la Teoría pura del Derecho de Kelsen ostentaba tal exigencia, Bobbio dedicó varios ensayos de defensa de tal postura contra los críticos marxistas y realistas. De esta defensa se puede obtener una primera aproximación a lo que se denominó: análisis estructural del ordenamiento jurídico; Bobbio señala que el enfoque de la "Teoría Pura" sigue sobresaliendo por su rigor, coherencia e integridad.

Después de 1955 la investigación de la Lógica Normativa o Deóntica avanzó, gracias a Kalinowski y Von Wright, así como las investigaciones sobre el mismo tema por parte de García Maynez que aportaron los nuevos conceptos lógicos y axiológicos acerca del manejo de las antinomias y la resolución de las lagunas, como efectivamente se presentan en la segunda parte de Teoría general del Derecho.

\section{GLOBALIZACIÓN Y DERECHO}

En la década del sesenta ocurrieron nuevos acontecimientos, que Bobbio reconoce como fecundos para el avance del Derecho; éstos son: la publicación de Law and Justice, de Alf Ross; la segunda edición revisada y aumentada de Reine Rechtslehre, de Kelsen, la cual supera la Teoría Pura del Derecho; The Concept of Law, de Herbert Hart. Estos libros aún hoy en día siguen siendo objeto de debate en el mundo occidental y en Europa Oriental. No obstante, la tesis kelseniana según la cual la Teoría de la Norma Jurídica lleva inevitablemente a la Teoría del Ordenamiento, con la consecuencia de que la definición de Derecho no puede ser dada, sino hasta que se parta del punto de vista del ordenamiento en 
su conjunto, también fue sostenida por el Profesor Herbert Hart, mediante de las, denominadas por él, "normas primarias y secundarias".

\section{EL ESTRUCTURAL FUNCIONALISMO EN LO JURÍDICO}

En el texto de Bobbio se distinguen las conceptualizaciones de estructura y función, pues en los estudios hechos hasta entonces sobre la naturaleza del Derecho se ubicaban en el terreno de la estructura, que es lo que se conoce como enfoque "formal" del Derecho. Pero, superada esta etapa, surgieron las inquietudes por el estudio y la profundización de los aspectos relacionados con la función del Derecho. Uno de los aspectos fundamentales que hacen que la concepción que se tenía en los años sesenta del Derecho perdiera relevancia, es la nueva orientación del Estado Social; según esta orientación el Derecho ya no sólo tiene la función negativa de proteger simplemente los Derechos Humanos y reprimir sus violaciones, sino también debe impulsar las acciones socialmente útiles, recurriendo a sanciones positivas (premios o incentivos), muy superficialmente consideradas en las teorías tradicionales.

Ahora bien, dicho cambio para el interés de los juristas de pasar de los temas estructurales a los funcionales fue consecuencia del trabajo interdisciplinario del Derecho con la Sociología, que cuestiona el formalismo imperante, que con aportes como los de Talcon Parson en los Estados Unidos (Estructural funcionalismo) y Niklas Luhmann (Teoría de los Sistemas) en Alemania, reaniman el trabajo integrado de Sociología, Derecho y Cibernética. En 1973 el Congreso Internacional de Filosofía del Derecho, celebrado en Madrid, se dedicó completamente al tema de la "función del derecho", en cuyo evento Bobbio, mediante un artículo escrito en memoria del filósofo jurídico argentino Ambrosio L. Giogia, contribuyó a dicho desarrollo funcionalista del Derecho.
Por otro lado, se aclara que los nuevos enfoques funcionalistas del Derecho, tan apreciados por los sociólogos, no reemplazaron los enfoques estructuralistas, sino que los complementan. Desde la perspectiva creadora de la dialéctica, los perfecciona, en relación con su estructura. Esto se puede observar cuando Bobbio afirma: "se ignora o se finge ignorar, por efecto del vicio común a toda escuela de tender al exclusivismo, que todo campo de la realidad puede ser considerado desde muchos puntos de vista, y que más bien esta multiplicidad de enfoques ayuda a su mejor comprensión" (Bobbio, 1992, p. XI). Una vez más, se demuestra la condición de pensador equilibrado y despojado de extremismos y parcializaciones que ostenta Bobbio.

Mi análisis personal acerca de la importancia no sólo del texto Teoría general del Derecho, sino también del conjunto de obras del mencionado autor y aún de los autores de la misma Escuela, se refiere a insistir que se incrementen los esfuerzos de la investigación ínter y transdisciplinaria, a fin de hacer de nuestra formación estudiantil verdaderos humanistas, eficaces en los procesos de transformación de estructuras, que conduzcan al logro de una verdadera democracia en todos los órdenes de la sociedad. Es evidente que los enfoques de esta escuela y en particular de Norberto Bobbio supieron establecer la íntima relación que existe entre el Derecho, como disciplina normativa, y la Ciencia Socio-Política como conjunto de aplicaciones de las Ciencias Sociales, así como de la Filosofía de la Historia, en los objetivos de la democracia, como el medio ordenador más racional y socio afectivo para lograr la igualdad y la Justicia.

\section{DERECHO, ESTADO SOCIAL Y DEMOCRACIA EN INTERACCIÓN}

Lo anterior está basado en la observación de la coherencia y la consistencia que existe entre la Teoría general del Derecho, la obra objeto del presente análisis, y el Futuro de la Democracia, texto del 
mismo autor. Asimismo, se observa consistencia lógica y metodológica con otra obra de Bobbio: La teoría de las formas de gobierno en la historia del pensamiento político.

\section{COMENTARIOS A LA PARTE PRIMERA}

\section{Acerca de la Teoría de la Norma}

Esta primera parte constituye el estudio y la profundización de la parte formal del Derecho, de los "continentes de contenidos", de las apariencias formales del Derecho, que permitieron pensar, hasta los años setenta, que el Derecho se podía identificar como disciplina o como ciencia, exclusivamente por su carácter normativo. Las grandes discusiones de los teóricos jurídicos, desde la época del imperio romano hasta Hans Kelsen, consistían fundamentalmente en el aspecto formal, es decir, en el carácter o la naturaleza de las normas, de lo cual devienen un número variado de teorías del Derecho, desde las escuelas moralistas hasta las escuelas positivistas estatalistas y las escuelas sociológicas y marxistas.

\section{Las clasificaciones de normas obedecen a diversas necesidades}

No obstante, tales teorías, basadas en dichos enfoques, sirvieron para establecer que definitivamente el Derecho no se podía identificar con el carácter normativo, identificación que, desde luego, se buscó siempre por su diferenciación con la moral y los convencionalismos sociales. También se podría agregar que sirvió para clasificar las normas; pero el derecho como la moral y los convencionalismos ostentan normas que por su estructura, es decir, por sus formas gramaticales o por sus expresiones sígnicas eran similares o idénticas, lo que arrojaba como resultado que los esfuerzos por encontrar la naturaleza de esta disciplina fueran nulos. El derecho, como la moral y los convencionalismos sociales contienen normas hipotéticas, normas imperativo categóricas, generales y particulares, abstractas y concretas, positivas y negativas, unilaterales y plurilaterales, de competencia, técnicas, organizacionales, de coordinación, mandatos, consejos, imploraciones, súplicas y peticiones, prescriptitas, descriptivas y expresivas, intersubjetivas, etc.

\section{Una concepción procesal del Derecho}

De lo anterior, se pudo concluir que, desde el punto de vista analítico, es evidente que el Derecho ha contribuido con el lenguaje en lo que se refiere a la búsqueda de mayores y más ponderados sistemas de comunicación, lo cual se ha revertido al Derecho mediante más sistemas de análisis de las formas comunicativas, como se observa con las contribuciones de la semiótica, la pragmática o la tagmética, por señalar algunos compartimentos investigativos de la lingüística, en general. Asimismo, tal interacción permanente ha servido a determinados enfoques de la lógica y de la filosofía del lenguaje, como aquélla de que el lenguaje, en su función de representación de los diversos enfoques de la realidad multivariable, ha permitido establecer aquella interacción que le permite señalar a Jurgen Habermas -filósofo y lingüista alemán, perteneciente a la Escuela de Francfort- en su obra Teoría de la acción comunicativa, que el lenguaje al foto ${ }^{1}$ permite que el mundo duro o mundo de la realidad natural externa a nosotros, mediante el lenguaje descriptivo, interactúe con el mundo de la realidad social, y ésta, mediante el lenguaje prescriptivo interactúe en los procesos de integración social, fundamentalmente; de igual forma, el mundo de las interioridades de las personas o mundo subjetivo, mediante el lenguaje expresivo, perfeccione la subjetividad y la personalidad de los sujetos, en general, y específicamente de los del derecho. Tales funciones son especialmente relevantes en el lenguaje jurídico, en el discurso

$1 \quad$ Reducir la realidad de los mundos que podríamos señalar, como hasta ahora compartidos universalmente por la generalidad de la sociedad. 
jurídico o la nueva Teoría de la argumentación (Habermas, 1987, pp. 104 y 309).

Por otro lado, se revierte de los estudios lingüísticos hacia el Derecho de manera positiva, las investigaciones de la pragmática y de la semiótica, según las cuales las intencionalidades de los hablantes o de los sujetos comunicantes se pueden inferir de las formas lingüísticas o simplemente simbólicas, vistas y analizadas en contextos específicos de comunicación ${ }^{2}$. La multiplicidad de normas con las que el Derecho trabaja y que resultaron de las múltiples réplicas y contra réplicas de las teorías formales alimentaron los esfuerzos investigativos lingüísticos en torno a las diferentes intensidades de fuerza con las que el lenguaje jurídico estructura las diversas relaciones intersubjetivas y de autoridad reguladora u ordenadora de las sociedades ${ }^{3}$.

Para los efectos prácticos del presente artículo, pienso que resultó clave a las explicaciones de comprensibilidad del Derecho, el aporte de Teorías de los sistemas, texto en el cual, a pesar de provenir del estudio de la Cibernética, se perfeccionaron los estudios biológicos y neuro sicológicos y resolvió muchas inquietudes de las Ciencias Sociales, específicamente con la Ciencia Jurídica.

\section{El Derecho como proceso, deviene en sistema}

Si bien Bobbio avanzó en las concepciones de los sistemas en la primera parte del estudio de la norma jurídica, las cuales estaban basadas en las aproximaciones de Hans Kelsen (sistemas estáticos y sistemas dinámicos), para referirse a la estructura de un primer precario ordenamiento y explicación de la clasificación de normas generales o abstractas hacia normas particulares o concretas, y normas

2 Aportes de la investigación interdisciplinaria entre Filosofía de la lengua y Sociolingüística (Escandell Vidal, 1993, p. 30).

3 Se recomienda consultar John R. Searle (1992) e Ignacio Izuzquiza (1990). apoyadas en el poder sancionatorio, y un segundo ordenamiento más complejo y relacionado con el pluralismo jurídico y la producción incesante del Derecho, en el que incluyó la lectura de normas de competencia, como normas técnicas necesarias para explicar que el poder de dictar normas emana de un primer principio soberano que le imprime el carácter de unidad al Derecho. Éste poder siempre tendrá límites, como uno de los principios reguladores de la concepción sistémica, que se empieza a estudiar y a revelar en las teorías estructural funcionalistas, aportadas inicialmente por Talcon Parsons y desarrolladas filosóficamente por su alumno Niklas Luhmann, quien le atribuyó al Derecho propiedades adicionales como ser "autoreferencial" $y$ "autocreativo" ("autopoiético"), lo que ya va a constituir característica fundamental de los "sistemas abiertos", similares a los sistemas de los organismos vivos (Niklas, 1990, p. 11).

\section{El Derecho sistema abierto de eficacia reforzada}

Antes de hablar de los sistemas, la primera parte de Bobbio sobre "La teoría de la norma jurídica" no está inconclusa en comparación con el Segundo libro "La teoría del ordenamiento jurídico", pues en la primera parte se llega a la demostración del hecho de que si la moral, el derecho y los convencionalismos sociales son todos normativos y ningún tipo de norma por su estructura formal, ni siquiera por la evidenciación semiológica de las intencionalidades del legislador moral, convencional o jurídico se llega a descubrir "per se" la naturaleza del Derecho, entonces es porque la naturaleza de la norma jurídica debe ser descubierta por el tipo de ordenamiento ("tipo de Sistema"), que sí tiene características propias que lo diferencian de los ordenamientos morales y convencionales de la sociedad.

Desde luego, en una de las teorías sobre la norma, Bobbio observa una intuición acerca de esta nueva y actualizada teoría del ordenamiento. Se trata de 
la Teoría Institucionalista, de Santi Romano, quien afirmó que primero fueron las instituciones y luego las normas, pues sin organización no pueden existir normas. Sin embargo, en el desarrollo del texto se muestran algunas de las inconsistencias de dicha teoría, similar a la discusión de "si fue primero el huevo o la gallina". Bien se podría decir que para que exista institución se requieren normas, entre otras cosas, porque toda norma es un principio de organización. Este enfoque fue desarrollado por el jurista argentino Carlos Cossio en su obra La valoración jurídica y la Ciencia del Derecho, en la que afirmaba que "toda norma era un orden".

En este momento es necesario abordar la segunda parte, aclarando y concluyendo de antemano, que la norma jurídica sólo se podrá describir e identificar como tal por el Ordenamiento Jurídico al cual pertenece, que dicho ordenamiento es sistémico, autoreferencial y autocreativo (autopoiético) (Izuzquiza, 1990; Von Bertalanffy, 1976, p. 47) y que el proceso de producción jurídica se desarrolla por virtud de su entorno social histórico resolviendo necesidades complejas de su época y de su tiempo y se adapta a los nuevos sucesos de las épocas de transformación, de manera similar a las operaciones y a la reproducción de los organismos vivos (Bobbio, 1992, p. 127).

\section{COMENTARIOS A LA SEGUNDA PARTE}

\section{De la teoría del ordenamiento jurídico}

El estudio de la primera parte corresponde a los elementos o componentes del sistema y ofrece pautas sobre las diversas modalidades y cómo se desarrollan las relaciones entre dichos elementos dentro del sistema, es decir, las diversas clases de relaciones de comunicación, como las normas, se entrelazan desde la perspectiva "estática" -en términos de Kelsen- $y$ desarrollan el programa de finalidades y de objetivos constitucionales, mediante subsistemas institucionales de desarrollo de diferentes Derechos Humanos.

Con lo anterior no se puede negar que su estudio muestra que dichas relaciones obran en una perfecta sincronía lógica, que ha dado a entender a muchos estudiosos que si bien no surge una nueva lógica jurídica como consecuencia de la concepción del sistema, la pluralidad de normas refleja la interacción con que el Derecho acoge la intersectación de los diversos mundos en los cuales se mueve la acción humana y sus intereses y valores más caros. En términos habermasianos lo anterior se refiere al mundo duro de la realidad externa al hombre, siempre en proceso de transformación por el trabajo humano, y del cual el hombre extrae permanentemente los recursos de su subsistencia y de su perfeccionamiento psico biológico; al mundo de sus interacciones sociales, socioeconómicas, comerciales, de intercambio material, valorativo, educacional y espiritual y de coordinación organizativa de poderes; $y$ finalmente al mundo interior 0 de la interioridad, que está conformado por selectas interacciones afectivas, sentimentales, estéticas y culturales (Habermas, 1987).

Tales mundos se van a reflejar en los lenguajes "descriptivo" o informativo, "prescriptivo" o direccional y "expresivo" o simbólico, poético o dramatúrgico, lenguajes, especialmente relevantes en la cotidianeidad de las normas. Estos lenguajes en el uso diario del Derecho vienen a formar una compleja estructura de relaciones que impulsan sistemáticamente los procesos, mediante los cuales el Derecho, en una estructura viva y hablante, organiza de forma permanente el desarrollo social, político y económico de una nación, en todas sus dimensiones. Es en este momento en que se puede observar la versión de sistema "dinámico", de Kelsen (Bobbio, 1992). 


\section{La consistencia teórico práctica del Derecho como sistema}

Las normas generales y abstractas desarrollan los principios de igualdad y la generalidad con la cual un pueblo entero se unifica y se proyecta en una expectativa común de comportamiento o de organización. Estas normas incorporan la expresión de la voluntad popular y el interés general (principios de igualdad), para que la sociedad entera desarrolle un programa político económico en el que los valores más universales, como los Derechos Humanos, no sólo sean respetados, sino también desarrollados gradualmente, con la potenciación de los recursos técnicos y científicos con que cuenta la Nación y la soberanía territorial.

Sin embargo, es necesario aclarar que ésta es la razón por la cual dictan normas, en su máxima expresión de la generalidad y la abstracción, como las que se encuentran en la Constitución Nacional y las cuales no pueden ser excluidas de su incorporación al Derecho nuevo, sino que, por el contrario, ellas cuentan de manera permanente en el proceso de desarrollo discursivo legislativo del Derecho y en los espacios y tiempos en que se concretan las normas, cada vez más particulares e individuales, mediante las "instituciones", que puntualizan su efectividad. En un Estado social de derecho la abstracción y la generalidad normativa encapsulan técnicamente la concreción de determinados valores universales, que la humanidad ha definido ya, después de grandes luchas sociales e históricas, y que constituyen, no ya una aspiración utópica, sino de realidad posible. Ya no se habla de la verdad como correspondencia con la realidad material, sino de la "verdad" como "camino". Es lo que Habermas llamó el mundo posible y factible (Habermas, 2005).

"Todos los hombres, de todas las razas y condiciones tiene que tener acceso real a la propiedad, la educación, la salud y el trabajo, por decir, lo mínimo". De ahí en adelante se desarrollaron el resto de Derechos Humanos, los cuales fueron incorporados a la constitucionalidad moderna de los países, sean éstos capitalistas o socialistas. Por otra parte, la democracia como forma jurídica que concretiza el derecho inherente a los ciudadanos de todos los pueblos, de participar no sólo con el voto, sino también con todas sus facultades comunicativas en la proyección de todos los recursos del desarrollo político, social y cultural se hace inherente al constitucionalismo y al derecho objetivo de los pueblos.

\section{La denominación "Derecho nuevo" corresponde a Derecho consistente. No se entiende el Derecho sin sus operadores}

El Derecho "nuevo" (Sáchica, enero-junio de 2007) es, por lo tanto, un derecho que tiene que ser desarrollado dentro de toda la lógica sistémica que incorpora; es decir, el sistema democrático de producción del Derecho, como una permanente construcción de la participación ciudadana, implica perfeccionamiento de las estructuras democráticas, como los partidos y los movimientos, y con mayor razón el Legislativo, sin las cuales el Derecho como tal encuentra frustraciones graves en su desenvolvimiento.

\section{La plenitud una necesidad del Sistema Jurídico, mientras la coherencia es un deber ser}

Cuando los jueces -especialmente los de las altas cortes- en sus procesos de interpretación sentenciales llenan los vacíos de la Ley o condicionan sus sentencias en desarrollo del llamado Nuevo Derecho, no lo hacen por desinstitucionalización del Derecho mismo, sino porque la Justicia, como aplicación del Derecho, no da espera, ni puede ser ilusoria, ni abstracta, y debe suplir subsidiariamente (para evitar la "entropía" del Sistema Jurídico) las fallas que se vienen dando por la deficiencia del Legislador, no acordes con el desarrollo de la nueva constitucionalidad. Al respecto se debe 
aclarar, como ya lo ha avizorado la Corte Constitucional en sus sentencias, la Constitución es Ley de la República y su vigencia no puede ser soslayada so pretexto de la Ley del Congreso (Corte Constitucional, sentencia C-083-95).

En una democracia institucionalizada, como es la que se busca con el Nuevo Derecho, la misión fundamental del Parlamento es proceder de manera inmediata y planificada a actualizar la ley con respecto a la constitución nueva en aras de la explicitez y no pretender que la ley preexistente a la Constitución prosiga incólume, porque entonces sería inocua toda reforma constitucional. El legislador debe renovarse y renovar la actualización de la Ley a nuevos principios marcados por ella en desarrollo del mandato de la soberanía popular, precisamente para evitar que tal función en los posible corresponda al Juez.

Desafortunadamente, históricamente, "el Legislador del interés general", al que hacía alusión Rousseau y en quien confiaba para la estructuración de la Ley, se extravió en los abismos de los intereses monetarios al ser elegido por minorías claramente agrupadas por intereses particulares, cuyos mandatos específicos constituyen la condición de su renovación en el cuerpo legislativo, dando lugar a una especie de burocracia legislativa, insensible al "interés general", especialmente para aquello que no les pueden aportar recursos en sus campañas de la democracia del voto. Esta situación puede y debe ser corregida en el futuro de la democracia, mediante el voto por programa, así como por el sometimiento de los partidos a estructuras técnicas, científicas y moralmente democráticas en estas infraestructuras políticas, cuya misión debe ser, constituirse en "venas de capilaridad social" que recojan en forma disciplinada $y$ universitaria la opinión y la formación de la voluntad popular, y organicen y reprogramen, para que a través de sus representantes formen la estructura legislativa parlamentaria acorde con el Derecho. Sólo así se cumple el ciclo sistémico de retroalimentación del entorno en la teoría de sistemas.

\section{El perfeccionamiento de la democracia necesidad del Derecho}

A pesar de que el legislador no le obliga la coherencia de la Ley con la Constitución por el carácter amplio que el elector posee para la elección de quienes integraran el legislativo (Bobbio, 1992), ya que la Constitución no exige que éstos sean profesionales del Derecho, expertos en lógica jurídica, si un mínimo de cultura jurídica debieran poseer los candidatos a dichas corporaciones como: la Cámara y el Senado, las Asambleas Departamentales y los Concejos Municipales, aun los Ediles. Es evidente que los ideales políticos e ideológicos de los partidos se deben concretar en estructuras jurídicas, organizacionales y administrativas, además de especificarse en las normas de contenidos materiales, por lo que se afirma que el principal creador del derecho es el legislador.

El hecho de carecer de formación jurídica y de formación en los procedimientos técnicos de redacción de estructuras formales legales, por parte de los integrantes del Congreso, es una de las falencias más graves para el desarrollo de la democracia y del Derecho. La adecuada construcción del lenguaje jurídico y de su sentido de justicia de primera fase, constituye una de las principales premisas de la paz; esto implica un lenguaje apropiado al entendimiento de la totalidad de los ciudadanos, es decir, un lenguaje transparente. No obstante, no sólo puede ser apropiado para el sector ilustrado de la población minoritaria. Un gran número de entuertos, incorporados a la legislación, han generado incertidumbre sobre la Ley y dificultades para su aplicación, tanto en lo que respecta a los Jueces como al Ejecutivo. Pero lo que es más grave para la democracia y para el Derecho es que a tales situaciones no se les pone empeño por las clases dirigentes, ya que en "río revuelto pescan los politiqueros y burócratas" 
que mediante una desviación del sistema jurídico inclinan la balanza hacia un "orden injusto", que nunca resuelve los problemas fundamentales de los ciudadanos y aplaza indefinidamente el anhelado proyecto libertario de la Justicia Social. Se trata pues, en adelante, de que se perfeccione el "Sistema".

El anterior ex-curso al comentario del segundo Libro de Bobbio, Teoría general del Derecho, lo he realizado para mostrar un poco la íntima relación que existe entre el Derecho y la democracia como medio político pertinente para lograr una sociedad civilizada y racional para la producción de los cambios sociales necesarios en la actualidad. Si se retoman las ideas que fueron concluyentes en la primera parte, el pluralismo normativo en el discurso jurídico, un aspecto que viene a ser determinante para el entronque con el ordenamiento, es el aspecto de la aplicación de la sanción, o más ampliamente del uso de la fuerza, para garantizar el orden y la aplicación efectiva de las normas jurídicas. La razón de lo anterior recae en que no todas las normas jurídicas tienen sanción, a menos que por sanción se entienda, no sólo la consecuencia negativa del incumplimiento de la norma, sino también la positiva, que bien se puede tratar de la indemnización en las normas civiles por fuerza de la obligatoriedad judicial o del estímulo para quien la cumpla, como empieza a darse con el beneficio tributario al empresario que desarrolle nuevos empleos, como efecto de la extensión lingüística del discurso jurídico en busca de la eficacia del derecho, propiedad del mismo, adicional a su validez y a su justicia.

\section{El Estado social de derecho, fuerza y consenso}

La historia del Derecho nos indica el "nuevo Derecho" que marcha paralelamente con la historia de las ideas políticas y del Estado, por lo que el Derecho como experiencia vivencial y social, nunca ha dejado de afirmar que el uso de la fuerza inherente al Estado no sirva para garantizar el cumplimiento de la Ley.

Los nuevos enfoques del Derecho señalan que la palabra Derecho Subjetivo, inherente a la personalidad jurídica se puede sustituir por la palabra "poder", para destruir una ambigüedad lógica, que ha hecho confundir el Derecho como lo "obligatorio". Pues bien, la fuerza, que desde luego no se puede confundir con la violencia, a pesar de cierta composición física y material, ha sido transformada históricamente por el carácter "cuasi mágico" del Derecho en un objeto cultural, si bien en épocas pasadas, dicha expresión las monarquías la fusionaron con el poder del monarca, con la tridivisión del poder comenzó la transformación de aquella expresión en fuerza cada vez más "apropiada", primero por las instituciones y luego por los individuos como sujetos.

En los tiempos modernos y posmodernos se habla de los Derechos Humanos, como "aquellos poderes" que le permiten a los seres humanos realizar más acciones y tener mejores y más reales opciones de elección. El poder de la vida, de la salud, de los recursos económicos, del conocimiento, de la tecnología, de la transportación, de la comunicación, etc. La norma tiene el poder mágico no sólo de prescribir mediante las obligaciones, sino también de atribuir facultades, permisos o poderes inscritos y descritos en los llamados Derechos Humanos, Derechos Subjetivos, Derechos Reales o Derechos Personales.

\section{El totalitarismo de Hegel y el pluralismo del Estado de derecho}

A partir de Hegel la historia del pensamiento humano comenzó a ubicar el conocimiento de la realidad como interacción de elementos múltiples que se relacionan entre sí en una totalidad, que viene a ser el límite de operaciones básicas que éstos desarrollan para una o algunas funciones específicas (Habermas, 1990, p. 123). 
Ortega y Gasset lo llamó "la historia como sistema". En ella el "espíritu absoluto" se desenvolvió en realidades históricas por un procedimiento eminentemente dialéctico y racional y culminó en la plenitud de las libertades humanas en el Derecho. Marx y Engels acogieron el concepto de sistema, pero lo ubicaron en un desenvolvimiento histórico material, en el que la razón humana encuentra explicación de las causas del desarrollo socioeconómico, a partir de los conflictos de clases y de intereses. Sin embargo, al mitificar dichos sistemas, estos autores se olvidaron de continuar el estudio de los sistemas en sí, que no constituyen una propiedad de determinados asuntos sociales o materiales, sino que los sistemas constituyen una Teoría y simultáneamente una metodología, que permite establecer de manera simplificada la observación con realismo de lo que significa el mismo movimiento material y social, como las realidades se presentan de manera por demás bastante complejas. Según Luhmann, simplificar la observación y explicación del movimiento de las cosas para descomponer y recomponer la realidad observada es la base fundamental de la Teoría de los Sistemas según Niklas Luhmann (1999).

\section{El nuevo orden mundial en perspectiva jurídica y democrática}

Finalmente, en la obra de Bobbio se muestra, con gran precisión científica y de modo pedagógico, la intersectación de los Sistemas Jurídicos nacionales con el orden jurídico naciente internacional que en la época de la globalización tiende a asumir el control mundial del desarrollo de la Paz duradera y la reubicación de los controles locales con nuevos subsistemas de desarrollo normativo más apropiados a dicha correlación.

\section{CONCLUSIÓN}

Por todas estas razones considero bastante apropiado el conocimiento inicial de la Teoría general del Derecho de la Escuela Italiana, como intro- ducción al estudio del Derecho, entre otras cosas, porque el nuevo estudiante del Derecho encuentra en él, la explicación inmediata y sin ambigüedades de la concepción modular, que sin lugar a dudas, es una concepción interdisciplinar del Derecho como medio de contribuir al Desarrollo Social, económico y cultural de manera democrática, creativa y proposicional.

\section{BIBLIOGRAFÍA}

Adriano, Ramón. (1986) Compendio de teoría general del derecho ( 1 a edición). Editorial Ariel.

Alchourron, Carlos E. y Bulygin. (1993) Introducción a la Metodología de las Ciencias Jurídicas y Sociales. Editorial Astrea.

Atienza, Manuel. (1998) Introducción al Derecho ( $1^{\text {a }}$ edición). México: Colección Doctrina Jurídica Contemporánea, Distribuiciones Fontamara.

Atienza, Manuel. (1995) Tras la justicia. Una introducción al Derecho y al Razonamiento Jurídico. Barcelona: Editorial Ariel S.A.

Biscaretti Di Ruffia, Paolo. (1996) Introducción al Derecho Constitucional Comparado (1 $1^{\text {a }}$ edición). México: Fondo de Cultura Económica.

Bobbio Norberto. (1984) Origen y fundamentos del poder político. México: Editorial Grijalbo S.A.

Bobbio, Norberto. (1992) Teoría general del Derecho. Editorial Temis.

Bobbio, Norberto. (1994) El futuro de la democracia. México: Fondo de Cultura Económica.

Bobbio, Norberto. (1996) La teoría de las formas de gobierno en la historia del pensamiento político. México: Fondo de Cultura Económica. 
Bobbio, Norberto. (1997) El problema del Positivismo Jurídico ( $5^{\text {a }}$ edición). Buenos Aires: Editorial Universitaria de Buenos Aires.

Cárdenas Patiño, Alberto y Beltrán, Héctor. (1990) Introducción a la Semiología ( $2^{a}$ edición). Bogotá: Universidad Santo Tomás.

Cossio, Carlos. (1954) La valoración jurídica y la ciencia del Derecho. Buenos Aires: Editorial Arayu. Buenos Aires. Editorial Temis-Distribuidor. 1954.

Dellepiane, Antonio. (1994) Nueva teoría de la prueba. Editorial Temis S.A.

Duverger, Maurice. (1994) Los partidos políticos. Colombia: Fondo de Cultura Económica.

Escandell Vidal, María Victoria. (1993) Introducción a la pragmática. Universidad Nacional de Educación a Distancia. Anthropos Editorial.

Foucault, Michel. (1994) Hermenéutica del sujeto. Ediciones La Piqueta.

Foncault, Michel. (1996) La verdad y las formas jurídicas. Barcelona: Editorial Gidisa S.A.

García Maynez, Eduardo. (1940) Introducción al estudio del Derecho. México: Editorial Porrúa S.A.

Habermas, Jürgen. (1990) Teoría y praxis (2a edción). Editorial Temis S.A.

Habermas Jürgen. (1998) Teoría de la acción comunicativa (Tomos I y II). Editorial Taurus.
Habermas, Jürgen. (2005) Facticidad y validez (4a edición). Editorial Trotta.

Izuzquiza Ignacio. (1990) "La Sociedad sin Hombres". Niklas Luhmann o la Teoría como escándalo. Editorial Anthropos.

Klug, Ulrich. (1990) Lógica jurídica. Editorial Temis.

Luhmann, Niklas. (1990) Sociedad y sistema: la anulación de una teoría ( $1^{\text {a }}$ edición). Ediciones Paidós.

Peña Ayazo, Jairo Iván. (1994) Wittgenstein y la crítica a la racionalidad. Universidad Nacional de Colombia. Ecoe Ediciones.

Rodríguez, César. (1994) La decisión judicial. El debate Hart-Dworkin. Universidad de los Andes. Nuevo Pensamiento Jurídico. Siglo del Hombre Editores.

Searle, John R. (1990) Actos de habla (3ª edición). Ediciones Cátedra.

Searle, John R. (1992) Intencionalidad. Editorial Tecnos S.A.

Van Dijik, Teun A. (1989) Estructuras y funciones del discurso (6a edición). Editorial Siglo XXI.

Von, Bertalanffy. (1994) Teoría general de los sistemas. México: Fondo de Cultura Económica.

Yorski, D.P. y otros. (1970) Lógica. Editorial Grijalbo S.A. 